\title{
COVID-19-related knowledge, risk perception, information seeking, and adherence to preventive behaviors among undergraduate students, southern Iran
}

\author{
Mohammad Rayani $^{1,2} \cdot$ Saba Rayani $^{3} \cdot$ Fatemeh Najafi-Sharjabad $^{1}$ (D) \\ Received: 2 March 2021 / Accepted: 12 June 2021 / Published online: 20 June 2021 \\ (C) The Author(s), under exclusive licence to Springer-Verlag GmbH Germany, part of Springer Nature 2021
}

\begin{abstract}
Globally, the novel COVID-19 has been recognized as one of the most important pandemics and devastating diseases in human history, with many deaths and morbidities. In the absence of effective treatment and limited supply of COVID-19 vaccine, people must adhere to recommended preventive measures. This study aimed to determine the level of COVID-19 knowledge, perceived susceptibility, perceived severity, and health information-seeking and preventive behaviors as well as associated factors with preventive behaviors in a sample of Iranian students at Persian Gulf University. Data was collected using an online structured and validated questionnaire. Descriptive statistics, the Mann-Whitney U, Chi-square, and Spearman correlation tests were applied to analyze data. The significance level was set at $\mathrm{P}<0.05$. In total, 98\% (319/325) of the participants completed the survey. A majority of participants were aware of the three main COVID-19 symptoms: fever, dry cough, and shortness of breath, while half of them were unaware of gastrointestinal problems caused by COVID-19. More than half of the subjects had a low perceived susceptibility and severity toward COVID-19. Most of the participants engaged in preventive behaviors and got COVID-19 information from social media and the Internet. Preventive behavior was associated with perceived susceptibility $(\mathrm{P}=0.015)$, perceived severity $(\mathrm{P}=0.014)$, and health information seeking $(\mathrm{P}<0.001)$ of individuals toward COVID-19. The results of our research can help health authorities develop health promotion programs for student populations by providing baseline data.
\end{abstract}

Keywords COVID-19 $\cdot$ Knowledge $\cdot$ Perception $\cdot$ Information seeking behavior $\cdot$ Prevention $\cdot$ Students

\section{Introduction}

Globally, SARS-CoV-2, the virus that causes COVID-19, has had a major effect on human health. The novel COVID-19 has been recognized as one of the most important pandemics and devastating diseases in human history, with many deaths and morbidities globally that happened daily since it arose in December 2019 and are still occurring these days in

Responsible Editor: Lotfi Aleya

Fatemeh Najafi-Sharjabad

f.najafi@bpums.ac.ir

1 Department of Public Health, Faculty of Health and Nutrition, Bushehr University of Medical Sciences, Bushehr, Iran

2 The Persian Gulf Tropical Medicine Research Center, The Persian Gulf Biomedical Sciences Research Institute, Bushehr University of Medical Sciences, Bushehr, Iran

3 Department of Psychology, Faculty of Literature and Humanities, Persian Gulf University, Bushehr, Iran
May 2021 (Wadood et al. 2020; World Health Organization 2021a). COVID-19 differs from its previous ancestor in that, in comparison to seasonal influenza, it is extremely transmissible and contagious (Liu et al. 2020). The virus spreads mainly from human to human through respiratory droplets of saliva or direct contact from the nose of an infected individual, contributing to the immense number of infected people (Cai et al. 2020). During the prodromal period, infected humans develop and reproduce large quantities of the virus in the upper respiratory tract, during which time they are normally active and can continue to perform their normal activities, contributing to rapid and widespread infections (Peiris et al. 2003).

On 6 May 2021, WHO reported that confirmed COVID-19 cases have exceeded 155,665,214 million globally, with over $3,250,648$ deaths World Health Organization (2021a). In Iran, from January 3 to 8 February 2021, there have been 2,610,018 confirmed cases of COVID-19 with 73,906 deaths World Health Organization (2021b). As the number of confirmed cases and deaths associated with the COVID-19 pandemic continues to rise across the world, most countries have 
adopted containment and prevention measures, requiring people to drastically change their lifestyles and reducing their exposure to the virus (Sheeran et al. 2014). Community collaboration in the implementation of health prevention measures, such as following WHO and other government agencies' orders and guidelines, is critical to slowing the spread of the virus and limiting the pandemic's long-term effects on public health (Mant et al. 2021). The extent to which these strategies are realistic, appropriate, and relevant to the general public is determined by a variety of factors, including people's perceptions of the infection's risk and negative health consequences (Sheeran et al. 2014).

The Health Belief Model (HBM) was one of the wellknown theories of health behavior. It was developed in the 1950s to better explain why people used or did not use preventive services provided by public health departments, and it has since expanded to address newer prevention and detection issues (e.g., mammography screening, influenza vaccines) Glanz and Bishop (2010). Research engaging with the HBM seeks to understand an individual's belief in their personal susceptibility to a disease, the severity of the impact an individual believes the disease may have on their life, perceptions of barriers to or benefits of engaging in a behavior, and an individual's conception of the consequences of taking particular actions (Rosenstock 1974). Here, we focus on the first two factors of the HBM, susceptibility and severity perceptions, since the greater the disease's perceived severity and the more vulnerable a person believes they are to it, the more likely they are to participate in prevention behaviors (Rosenstock 1974; Rosenstock et al. 1988).

It is critical to comprehend people's disease risk perceptions (perceived susceptibility and severity) and how they influence the adoption of healthy behaviors. People's perceptions of their susceptibility to illnesses play a key role in deciding whether or not to take precautions. The subjective probability of developing an infection or finding oneself in a condition with a negative health result is reflected by perceived susceptibility (Rosenstock 1974). People who do not believe they are at risk of being ill are less likely to take care, putting themselves and others in danger Venema and Pfattheicher (2021). According to the HBM, perceived severity refers to an individual's subjective assessment of a disease's seriousness, which is influenced by a variety of factors like current reality and expectations for future events (Rosenstock 1974; Rosenstock et al. 1988). Weinstein (2000) showed that a high perception of disease severity leads to proactive health-protective behaviors (Weinstein 2000).

As one of the most dynamic groups, university students have great mobility and they like socializing. They are healthy, young, and often have mild symptoms after being infected with COVID-19, which can have a major impact on the spread of COVID-19 (Ding et al. 2020). Young adults' perceptions as a subset of the general population are crucial to understand because they have less comorbidities and less overall health concerns, resulting in a vastly different understanding of disease risk than older adults (Wickman et al. 2008). According to Ramsey and Marczinski (2011), college students "underestimate their risk level" (Ramsey and Marczinski 2011). In the situation of COVID-19, Faasse and Newby (2020) Australian research discovered that younger age (18-29 years) was linked to lower involvement in health-promoting behaviors Faasse and Newby (2020).

Individuals' information-seeking behavior during decision-making can have a major effect on decision outcomes Kolarić and Stričević (2017). Wilson defines information-seeking behavior as the purposeful seeking of information in order to achieve a specific objective. When searching for information, people may use either manual or computer-based information systems (Wilson 2000). According to Marchionini, information seeking is a basic human process that is linked to learning and problem solving (Marchionini 1997). In other words, when a person requires new information for some reason, he or she engages in deliberate or purposeful information seeking. An individual interacts with various information sources during this process Kolarić and Stričević (2017).

The ways in which people seek information about their health, risks, diseases, and health-protective behaviors are referred to as health information-seeking behavior (HISB) (Lambert and Loiselle 2007). An individual's HISB may be influenced by their health perceptions, current health status, and family health background (Jacobs et al. 2017).

Health information seeking is described by Lambert and Loiselle (2007) as "the type and amount of health-related information sought, the specific actions implemented to obtain the information, and the sources individuals use" (Lambert and Loiselle 2007). Individuals who engage in health information-seeking are more likely to provide improved health information, feel more relaxed and assured when talking to a doctor about health issues, and show higher levels of health promotion practices than individuals who do not seek health information (Shieh et al. 2010).

Although the importance of information seeking in decisionmaking is obvious, there has been little research on youth health information-seeking behavior, particularly among college students, in relation to COVID-19 during this pandemic.

Since COVID-19 continues to spread at an alarming rate, a basic study of university students' risk perception is needed. Students with insufficient risk perception and prevention behavior can underestimate the situation, reduce fear and anxiety, and disrupt appropriate prevention measures (Mant et al. 2021). A research gap exists regarding the extent of risk perception in response to health pandemics among university students. Therefore, this study aimed to determine the level 
of COVID-19 knowledge, perceived susceptibility, perceived severity, health information seeking, and preventive behaviors as well as associated factors with preventive behaviors in a sample of Iranian students at Persian Gulf University (PGU). The findings will contribute to better understand how students perceive danger and seek information in an emergency situation like the COVID-19 pandemic. The findings of this study represent the baseline levels of information that must be collected during scientific research in order to establish and implement evidence-based health behavior interventions and policies to mitigate the impact of the COVID-19 pandemic and identify methods for strengthening societal cooperation and responses to potential pandemic situations.

\section{Methods}

\section{Study design, setting, and participants}

A cross-sectional study was conducted from September to October 2020 among undergraduate students at Persian Gulf University (PGU), southwest of Iran. All potential population who studied at PGU were invited to participate in this study. The inclusion criteria were studying at PGU and willing to give informed consent. The exclusion criteria of the participants were as follows: questionnaire completion time less than $2 \mathrm{~min}$, incomplete completion, duplicate completion of the questionnaire with similar IP address, and basic information.

\section{Sample size}

Undergraduate students who are studying at PGU were the target population. The sample size was calculated using a single proportion formula. Based on a previous study done by Taghrir et al. (2020) among medical students in Shiraz, south of Iran, the proportion of high risk perception toward COVID-19 was 31\% (Taghrir et al. 2020). Taking the precision of 0.05 with $95 \%$ confidence, the sample size was calculated as 328 .

\section{Procedure}

A convenience sampling technique was used to recruit participants. An online structured questionnaire was provided by using Porsline software in Persian language. The link of the questionnaire was forwarded to the students by representatives of each class through WhatsApp. An online informed consent was obtained from all participants prior to their involvement in the research. Those who agreed were allowed to complete an online Web-based questionnaire. The participants' names were not recorded to assure confidentiality.
Also, written permission to use the questionnaires was obtained from all the authors via email.

\section{Instruments}

The structured questionnaire consisted of five parts:

\section{Demographic characteristics}

This section include age and gender of participants

\section{COVID-19-related knowledge}

Knowledge about clinical symptom of COVID-19 was assessed with seven questions and adopted from Shahnazi's study which was done north of Iran during the early period of the COVID-19 outbreak (Shahnazi et al. 2020). Each question was answered using "Yes, No, I don't know" format. Respondents were given 1 point for answering correctly and no points for answering wrongly or do not know. We computed a total COVID19-related knowledge score by summing the correct answers to the questions ranging between 0 and 7 .

\section{Risk perception toward COVID-19}

The risk perception of participants was assessed by two sub-scales including perceived susceptibility (3 items) and perceived severity (3 items), which was adopted from Shahnazi's study (Shahnazi et al. 2020). The item was scored using a Likert-type response scale ranging from 1 "strongly agree" to 5 "strongly disagree." The total score for COVID-19 perceived severity and perceived susceptibility ranged from 5 to 15 . Respondents' perceived severity and perceived susceptibility scores were categorized as follows: poor, if they obtained $<50$ th percentile, moderate for 50 th -75 th percentile, and high for $>75$ th percentile.

\section{Health information-seeking behaviors}

Health Information-Seeking Scale (HISS) measured health information seeking from multiple sources (Das 2013) (Feinberg et al. 2015). Seven items were included in this scale that respectively asked how often in the past month the students had looked for COVID-19-related health information from different sources, which include (1) printed sources (brochure, booklet, pamphlet); (2) health care providers (doctors, nurses, health workers); (3) national media (TV and radio); (4) international media (satellite); (5) Internet resources (websites, blogs, Internet newspapers); (6) social networks (WhatsApp, Telegram, Instagram); (7) family members, relatives, and friends. Responses in the HISS were coded from 1 to 4 , 
corresponding with never, sometimes, often, and all the time. The total score for HISS ranged from 7 to 28 .

\section{Preventive behaviors against Covid-19}

We selected nine types of preventive behaviors from the COVID-19 prevention guideline provided by World Health Organization World Health Organization (2021a, b). The COVID-19 preventive behaviors were measured with eight statements on a 5 -point Likert scale $(1=$ never, $2=$ rarely, $3=$ sometimes, $4=$ often, $5=$ always $)$. We calculated mean scores for these nine items, of which higher scores represented more engagement in preventive behaviors. The preventive behaviors included 1) staying home as much as possible, 2) wearing a mask when going out, 3) washing hands frequently, 4) avoiding public transportation, 5) avoiding party gathering, 6) social distance at least $1 \mathrm{~m}, 7$ ) trying to eat healthy and wellbalanced diets, 8) getting plenty of sleep, and 9) exercising regularly. Total score for preventive behaviors ranged from 8 to 40 .

\section{Validation and reliability of the scales}

The questionnaire was face validated by four specialists' opinions in the field of health educations and community health. After minor modifications, the questionnaire was forwarded to the participants. In the present study, the reliability measure using Cronbach's alpha coefficient was 0.67 for COVID-19 knowledge, 0.62 for perceived susceptibility and 0.64 for perceived severity, 0.70 for information seeking, and 0.74 for COVID-19 preventive behavior. All values of Cronbach's alpha for description of internal consistency were in good $(0.7 \geq \alpha \geq 0.9)$ and acceptable $(0.6 \geq \alpha \geq 0.7)$ ranges (Hulin et al. 2001).

\section{Outcome variable}

Outcome variable of this study was COVID-19-preventive behavior.

\section{Independent variables}

In this study, we considered the age, gender, knowledge, perceived susceptibility and perceived severity toward COVID19 , and health information-seeking behaviors as independent variables.

\section{Statistical analysis}

All the data were analyzed using IBM SPSS Statistics version 22. Descriptive analysis included frequencies and percentages, means, and standard deviations. The
Kolmogorov-Smirnov test was performed to determine the normality of the data, and the findings revealed that not all variables were normally distributed, based on the demographic characteristics $(\mathrm{P}<0.05)$. Therefore, nonparametric Spearman correlation test was applied to measure the strength and direction of association between two continuous variables. The Mann-Whitney U test was used to compare differences between two independent groups when the dependent variable is either ordinal or continuous, but not normally distributed. The chi-square test was used to determine associations among categorical variables. The significance level was set at $\mathrm{P}<0.05$.

\section{Results}

\section{Participants' characteristics}

Out of 325 participants who responded the questionnaires, six questionnaires were excluded because of incomplete answer to the questions. The mean age of the participants was $21.74 \pm$ 3.79 in the range of 18 to 50 years and $73 \%$ of the subjects were female.

\section{COVID-19-related knowledge}

The mean knowledge score was $4.75 \pm 1.25$ in the entire study group. The participants' responses to each knowledge questions about clinical symptom of COVID-19 have been shown in Fig. 1. A majority of participants were aware of the three main COVID-19 symptoms: fever, dry cough, and shortness of breath. Furthermore, approximately three-quarters of the students were aware of the symptom of muscle pain, while a majority of participants were unaware of the symptoms of a headache and a runny nose. Almost half of the participants were unaware that gastrointestinal problems like diarrhea and vomiting could be caused by COVID- 19 .

\section{Perceived susceptibility and severity of COVID- 19 infection}

The mean perceived susceptibility and perceived severity were $12.18 \pm 2.58$ and $8.87 \pm 1.93$ respectively. The level of respondents' risk perception on COVID-19 has been reported in Table 1.

More than half of the subjects had a low perceived susceptibility, and $12 \%$ had a moderate and $26 \%$ had a high perceived susceptibility toward COVID-19. Furthermore, $60 \%$ of participants had low perceived severity and $40 \%$ had high perceived severity toward COVID-19. 
Fig. 1 Distribution knowledge of clinical symptoms of COVID-19 among students $(\mathrm{n}=319)$

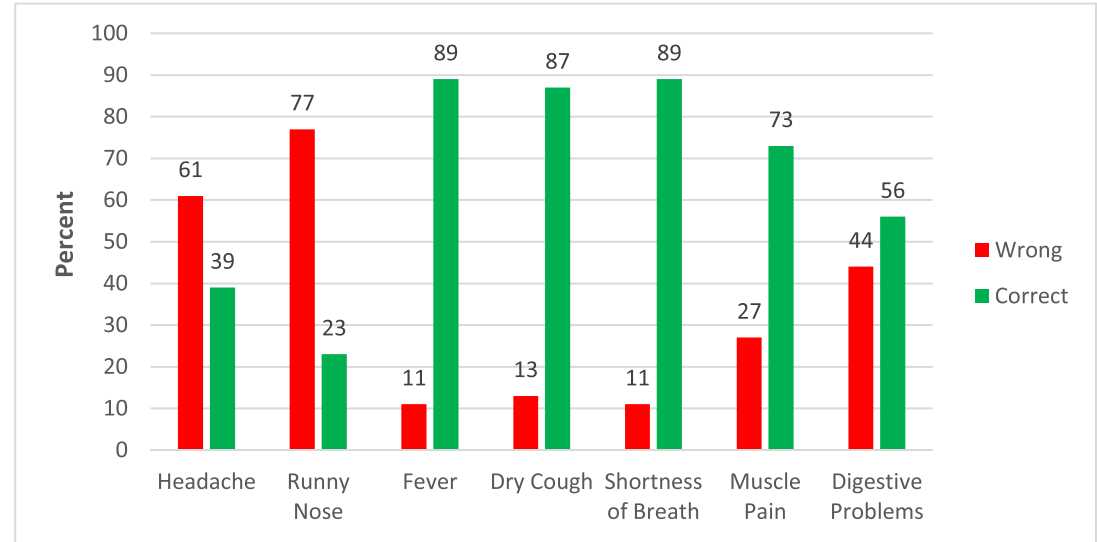

\section{Health information-seeking behaviors related to COVID-19}

The mean health information seeking score was $17.10 \pm 3.84$ in the entire study group. Figure 2 shows how respondents sought health information from various sources. The most commonly used sources for information about COVID-19 were the Internet (e.g., websites, blogs, Internet, and newspapers) and social media (e.g., WhatsApp, Telegram, and Instagram). The majority of participants rarely used health care professionals or the print media to look for information about COVID-19.

\section{Preventive behaviors against COVID-19}

The mean COVID-19 preventive behaviors score was $37.10 \pm$ 5.43. The frequency of participants' engagement in preventive behaviors against COVID-19 is reported in Table 2. Although participants largely engaged in all preventive behaviors, wearing a mask, washing hands, avoiding public transportation, and avoiding party gathering were the most frequently practiced preventive behaviors while exercising regularly and getting plenty of sleep were the least (Table 2).

\section{Factors associated with COVID-19 preventive behaviors}

There was a significant association between gender and preventive behaviors, indicating female participants were

Table 1 Level of perceived susceptibility and perceived severity of COVID-19 among students ( $\mathrm{n}=319)$

\begin{tabular}{llll}
\hline COVID-19 risk perception & Low & Moderate & High \\
N $(\%)$ & N $\%)$ & N $(\%)$ \\
\hline Perceived susceptibility & $198(62)$ & $38(12)$ & $(26) 83$ \\
Perceived severity & $(60) 191$ & 0 & $(40) 128$ \\
\hline
\end{tabular}

more likely to engage in COVID-19 preventive behaviors when compared to males (Mann-Whitney $\mathrm{U}=7405$, $\mathrm{P}<0.001)$. Figures 3 and 4 show COVID-19 preventive behaviors among male and female students respectively. Chi-square tests indicated female students more often/ always stay at home $(85 \%$ vs $64 \%, \mathrm{P}<0.001)$, wear a mask (93\% vs $84 \%, \mathrm{P}=0.009$ ), avoid party gathering ( $89 \%$ vs $78 \%, \mathrm{P}=0.016)$, and wash hands frequently ( $92 \%$ vs $80 \%$, $\mathrm{P}=0.003$ ) as compared to male students.

The Spearman correlation test showed that there was a weak but significant positive relationship between preventive behavior and perceived susceptibility $(\mathrm{r}=0.211, \mathrm{P}=0.015)$ and perceived severity $(\mathrm{r}=0.214, \mathrm{P}=0.014)$ of COVID-19 infection. This indicates that subjects with higher perceived susceptibility and severity of COVID-19 infection are more likely to engage in preventive behavior. Furthermore, preventive behaviors were significantly correlated with health information-seeking behaviors $(\mathrm{r}=0.0 .412, \mathrm{P}<0.001)$. This result indicates that with the increase of health informationseeking behaviors, preventive behaviors against COVID-19 also increase. The correlation coefficient between preventive behavior and health information seeking was 0.412 , which indicates a moderate level of correlation. No significant relationship was found between knowledge and preventive behaviors $(\mathrm{P}=0.464)$. The result of the Spearman correlation test is presented in Table 3 .

\section{Discussion}

The aim of this study was to determine the level of knowledge, perceived susceptibility, perceived severity, health information-seeking, and preventive behaviors toward COVID-19 as well as associated factors with preventive behaviors among students at PGU. This study was carried out in the first 6 months of the COVID-19 pandemic south of Iran. With the exception of regular exercise, most participants reported multiple preventive behaviors against COVID-19. Wearing a mask and washing hands were the most frequently reported 
Fig. 2 Health informationseeking behaviors related to COVID-19 among students

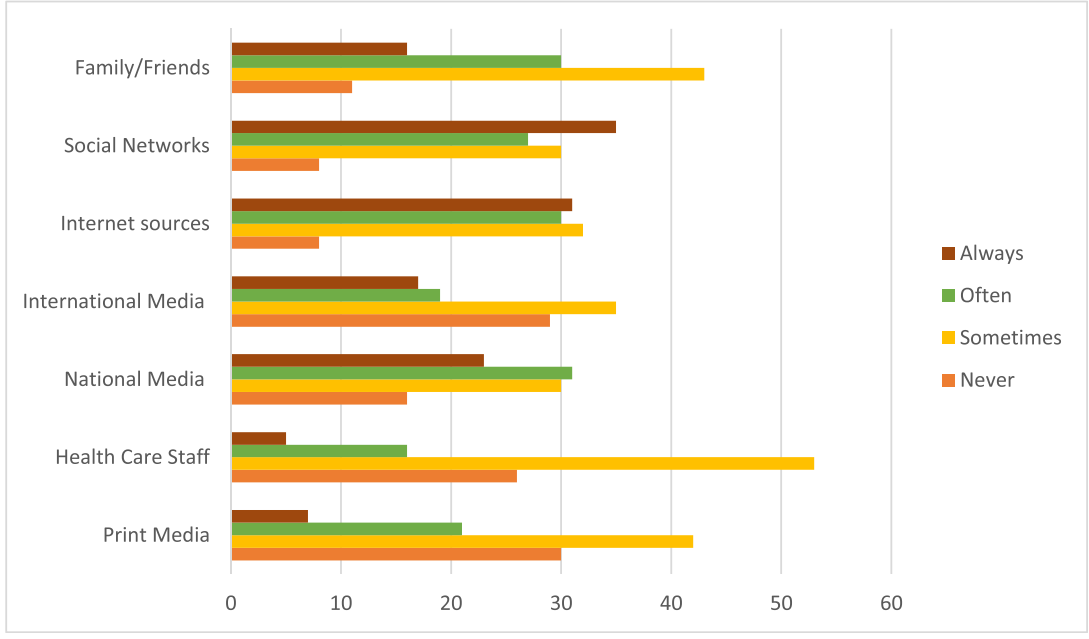

COVID-19 preventive behaviors, with $90 \%$ of participants saying they often/always wear a mask when going out and $84 \%$ saying they often/always wash their hands. Female individuals were more likely to engage in recommended health behaviors. This finding is consistent with previous researches on SARS and H1N1, which found that females were more likely to engage in health-related behaviors (Fung and Cairncross 2007; Lau et al. 2009; Liao et al. 2010; Rubin et al. 2009). Females were also more likely than males to claim they were able to self-isolate, according to the COVID-19 survey conducted in Canada and the UK (Atchison et al. 2020; Mant et al. 2021).

In this study, most respondents knew three main symptoms of COVID-19 including fever, dry cough, and shortness of breath, while their knowledge of other clinical symptoms like gastrointestinal, runny nose, and headache was low. In contrast, Jordanian university students demonstrated a high level of knowledge about clinical symptoms of COVID-19 (Alzoubi et al. 2020). The inadequate knowledge among the students reflects a lack of relevant education and may compromise their awareness of personal risk perception of COVID-19 infection and the severity of its consequences.

It is not self-evident that recommended precautionary behaviors would be adopted [6]. As a result, it is critical to pay special attention to the factors that influence behavioral change during infectious disease outbreaks (De Zwart et al. 2009). Effective public health programs that help people preserve and improve their health, minimize disease risks, and treat illness also require behavior change on multiple levels (e.g., individual, organizational, and community). Understanding health behaviors and the contexts in which they occur is the foundation of the most effective public health programs and initiatives. Models of strategic planning offer a systematic framework for designing and managing public health interventions, as well as evaluating and strengthening them. Health behavior model can help in program design and assessment, as well as development of new intervention strategies Glanz and Bishop (2010). Individuals and the community must learn and follow the appropriate behaviors in order to achieve healthy lifestyle practices and disease prevention. Risk perception is
Table 2 Frequency of participants' engagement in preventive behaviors against COVID-19 $(n=319)$

\begin{tabular}{lllll}
\hline COVID-19 preventive behavior items & $\begin{array}{l}\text { Never/ } \\
\text { rarely } \\
\mathrm{N}(\%)\end{array}$ & $\begin{array}{l}\text { Sometimes } \\
\mathrm{N}(\%)\end{array}$ & $\begin{array}{l}\text { Often } \\
\mathrm{N}(\%)\end{array}$ & $\begin{array}{l}\text { Always } \\
\mathrm{N}(\%)\end{array}$ \\
\hline Staying home as much as possible & $20(6.3)$ & $50(15.7)$ & $(29.2) 93$ & $156(48.9)$ \\
Wearing a mask when going out & $(2.8) 9$ & $(7.2) 23$ & $(10.7) 34$ & $(79.3) 253$ \\
Washing hands frequently & $(2.4) 11$ & $(11.3) 36$ & $65(20.4)$ & $207(64.9)$ \\
Avoiding public transportation & $(11.9) 38$ & $(13.8) 44$ & $(10.7) 34$ & $(63.6) 203$ \\
Avoiding party gathering & $(3.2) 10$ & $(13.5) 43$ & $(34.5) 110$ & $(48.9) 156$ \\
Social distancing at least $1 \mathrm{~m}$ & $(1.6) 5$ & $(10.7) 34$ & $(22.3) 71$ & $(65.5) 209$ \\
Trying to eat healthy food & $(3.5) 11$ & $(13.5) 43$ & $(40.4) 129$ & $(42.6) 136$ \\
Getting plenty of sleep & $(5.6) 18$ & $(25.7) 82$ & $(26) 83$ & $(42.6) 136$ \\
Exercising regularly & $(25) 80$ & $(41.7) 133$ & $(17.2) 55$ & $(16) 51$ \\
\hline
\end{tabular}


Fig. 3 COVID-19 preventive behaviors among male students

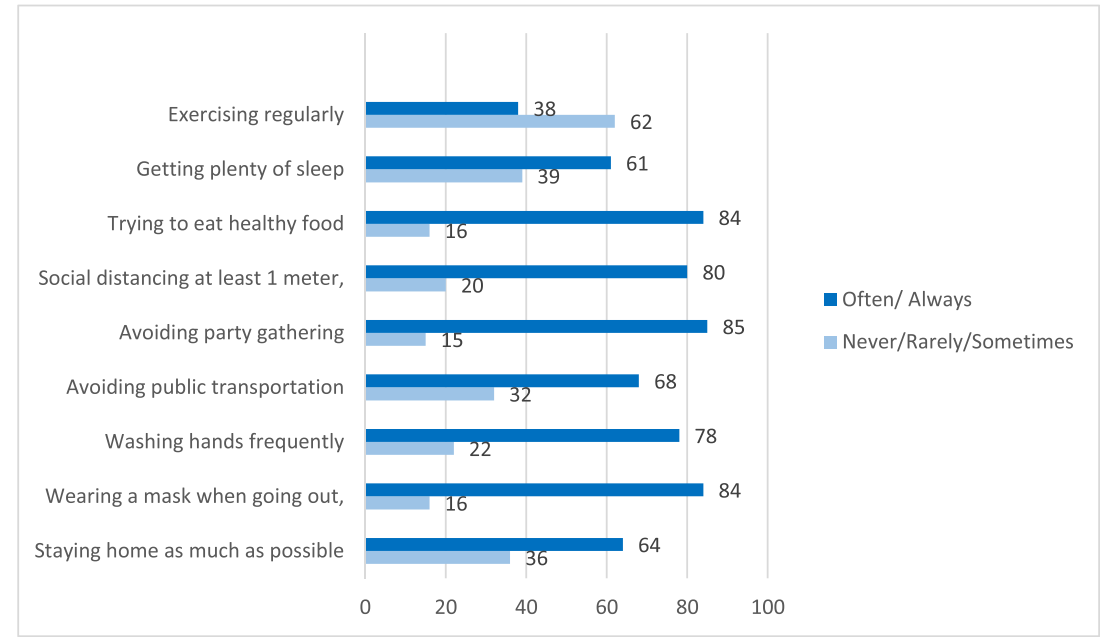

one of the factors that can influence people's willingness and motivation to take precautionary measures.

The HBM, which is the first theory relevant to health behavior, can explain preventive behavior and individual disease response Glanz and Bishop (2010). HBM is the origin of risk perception. The risk perception is a field of psychology that deals with a person's perception and understanding of different objective dangers in the environment. Risk perception is an important factor influencing risk behaviors. People who have a low risk perception are more likely to engage in risky behaviors or minimize preventive behaviors, while those who have a high risk perception are more likely to engage in preventive behavior (Ding et al. 2020). Risk perception and its predicates (perceived susceptibility and perceived severity) make the HBM well-suited to studying or modifying behaviors that may contribute to negative health outcomes. In the $\mathrm{HBM}$, an individual is thought to be more likely to take appropriate action if the disease's risk perception is high. In turn, if the disease is regarded as a serious problem, the risk perception will be greater. Similarly, risk perception will be increased if perceived susceptibility is increased, implying that the adverse outcome is regarded as something the individual could reasonably expect to experience Sheppard and Thomas (2021). Individuals who believe they are at low risk of developing a COVID-19 are more likely to engage in unhealthy or risky behaviors such as not wearing a face mask and being unable to maintain social distance (Teh et al. 2019).

Over half of the students in our study had a low level of perceived susceptibility and severity to COVID-19 infection. Y1ldırım and Güler (2020) discovered that the Turkish general population had high levels of COVID-19-related severity, and the majority of participants believed that contracting COVID19 would be extremely dangerous for them Yildirim and Güler (2020). During the COVID-19 pandemic, Shahin and Hussien (2020) conducted a study among the general population of Middle Eastern countries such as Saudi Arabia, Egypt, and Jordan. They discovered that Saudi Arabian participants had higher mean scores for the perception of COVID-19 disease susceptibility and severity than Egyptian and Jordanian participants. Egyptian participants were significantly less
Fig. 4 COVID-19 preventive behaviors among female students

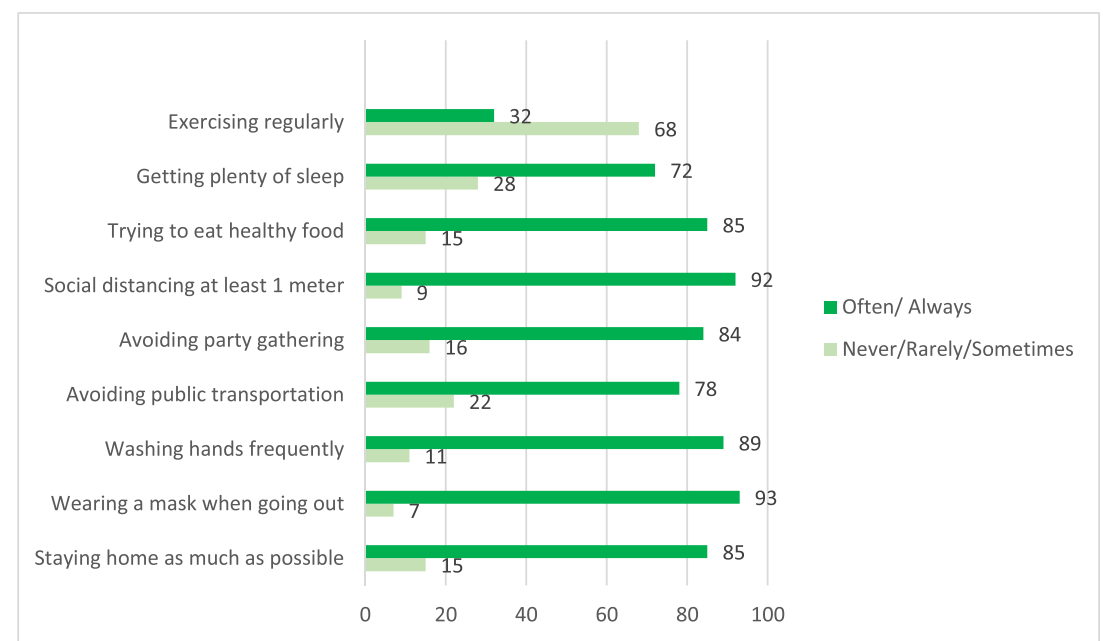


Table 3 Correlation between COVID-19 preventive behavior and knowledge, perceived susceptibility, perceived severity, health information seeking

\begin{tabular}{|c|c|c|c|}
\hline Correlation & $\mathrm{N}$ & $\mathrm{r}$ & P-value \\
\hline $\begin{array}{l}\text { Knowledge } \\
\text { Preventive behavior }\end{array}$ & 319 & 0.042 & 0.464 \\
\hline $\begin{array}{l}\text { Perceived susceptibility } \\
\text { Preventive behavior }\end{array}$ & 319 & 0.211 & 0.015 \\
\hline $\begin{array}{l}\text { Perceived severity } \\
\text { Preventive behavior }\end{array}$ & 319 & 0.214 & 0.014 \\
\hline $\begin{array}{l}\text { Health information seeking } \\
\text { Preventive behavior }\end{array}$ & 319 & 0.412 & $<0.001$ \\
\hline
\end{tabular}

likely than other populations to comply with COVID-19 precautionary measures. These disparities in risk perceptions may be due to the low number of confirmed COVID-19 cases and deaths reported in Jordan compared to Saudi Arabia and Egypt, which may influence participants' perceptions of seriousness. Furthermore, Saudi Arabia has previously experienced outbreaks of epidemic pathogens such as SARS and Ebola, which may serve as a base point for assessing risk perceptions of the current COVID-19 pandemic Shahin and Hussien (2020). The findings of our study contradicted the research results of Mants' study, which found that $96.5 \%$ of Canadian university students rated COVID-19 as moderately or very severe (Mant et al. 2021). The disparity in risk perceptions could be attributed to differences in the study population, study period, data collection tool and procedures, and the level of virus spread across countries and communities (Tadese et al. 2021).

In this study, perceived susceptibility to COVID-19 infection and perceived severity of health-related consequences were both linked to engagement in disease-preventive behaviors. This result was consistent with the findings of Bish and Michie, who found that higher perceived personal risk predicts an individual's participation in disease prevention behaviors such as hand washing and social distancing, as evidenced by studies of previous pandemics Bish and Michie (2010). College students, as a high-knowledge group, can not only serve as the foundation of future national construction, but they can also disseminate their knowledge and strong risk perception to those around them (Chandrashekharayya et al. 2014). As a result, the risk perception level of college students on COVID-19 is crucial to consider. Underestimation of this health risk may result in risk behaviors and neglect of early COVID-19 symptoms. Given the high infectivity of COVID-19 and the disease's occult existence (Liu et al. 2020) (Wang et al. 2020), a high proportion of low-risk perception of COVID-19 could lead to recurrent outbreaks.

People in modern society are expected to actively manage their own health and make a variety of health-related decisions. Several studies have found that individuals' protective health behaviors are influenced by their exposure to health information on social media (Namkoong et al. 2017; Yang and $\mathrm{Wu} 2021$; Lee 2011). The current study revealed that the vast majority of participants got COVID-19 information from social media and the Internet. There was a significant positive correlation between health information-seeking behavior and COVID-19 prevention behavior in this study. Greater compliance with recommended preventive behaviors was associated with a higher level of health information seeking toward COVID-19. This may be related to higher health literacy of the study sample. Ishikawa stated that those with higher levels of health literacy used newspapers and the Internet more frequently and were more likely to adopt protective measures against health risks (Ishikawa et al. 2016). Yang and $\mathrm{Wu}$ (2021) discovered that Chinese people who are exposed to health information on social media platforms such as Weibo are more likely to be aware of the dangers posed by air pollution and how to properly protect themselves, such as by wearing a PM2.5 mask, which contributes to their increased positive attitude toward this behavior (Yang and $\mathrm{Wu}$ 2021). Namkoong et al. (2017) found that increased social media use positively affected individuals' attitudes and perceived social norms about smoking behaviors, eventually reducing smoking intention (Namkoong et al. 2017).

Since all participants in this study were university students and Internet users, the use of Internet sources and social media as the most frequent source of health information is to be expected. As young people increasingly use the Internet and social media to obtain health-related information, it is critical to evaluate the quality of information, such as its accuracy, validity, and understandability (Esmaeilzadeh et al. 2018). Providing credible, simple, and understandable health information content by health information providers, especially medical librarians, is needed.

This study is based on a PGU survey conducted in southern Iran in response to the COVID-19 pandemic. As a result, this dataset serves as an important foundation for future comparative work. As the pandemic progresses, further research on individuals' health behaviors over time is required. Even as vaccines become more widely available, individual behavior in maintaining a flattened curve will be critical.

This study has several limitations. One can refer to the cross-sectional nature of the research, which made it difficult to establish a causal relationship between the determinant factors and COVID-19 preventive behavior. The study is based on a convenience sample of university students who chose to participate in the study on their own. The data was gathered solely through participant self-report. Self-report measures may have limitations due to biases such as social desirability and introspective ability. Because the study population was drawn entirely from one university, generalizations about other university contexts or the Iranian population may be inappropriate. Further research at other Iranian and international 
universities is needed to understand potential variability in student responses to the pandemic. Despite the dataset's limitations, our study provides insight into young adults' perceptions of COVID-19 and the health behaviors that resulted during the first 6 months of the pandemic.

\section{Conclusion}

As COVID-19 spreads relatively quickly through direct human-to-human contact, governments have been required to inform the public of the risks and necessary precautions for protecting themselves and others. In the absence of effective treatment for infected individuals or an adequate licensed vaccine to achieve global control of COVID-19, people must drastically alter their lifestyles and adhere to recommended preventive measures to mitigate the spread. Social and behavioral theories and models not only help explain health-related behavior and its determinants, but they can also guide the development of interventions to influence and change health-related behavior and ultimately improve health. In this study, we used HBM as a theoretical framework to explain COVID-19 prevention behaviors. A majority of participants were aware of the three main COVID-19 symptoms: fever, dry cough, and shortness of breath, while half of them were unaware of gastrointestinal problems caused by COVID-19. A low level of risk perception of COVID-19 was identified among university students. Most of the participants engaged in preventive behaviors and got COVID-19 information from social media and the internet. Females were more likely than males to engage in recommended health behaviors. This study demonstrates that preventive behavior was associated with perceived susceptibility, perceived severity, and health information seeking of individuals toward COVID-19. This result is particularly key given its occurrence in young adults, a subgroup known to have a lower baseline rate of health concerns than older adults. The results of our research can help health authorities develop theory-based health promotion programs for student populations by providing baseline data. Health promotion programs can be incorporated into future curricula to improve infection control knowledge.

Acknowledgements The authors appreciate the Vice-Chancellor of Research, Persian Gulf University, Bushehr, Iran for the collaboration and permission of this research. We also acknowledge the students at Persian Gulf University for their participation in this study.

Author contribution Fatemeh Najafi-Sharjabad: conceptualization, study design, statistical analysis, writing original draft preparation. Mohammad Rayani: manuscript writing review and editing, Saba Rayani: data collection. All the authors have read and agreed to the published version of the manuscript.
Data availability All data generated or analyzed during this study are included in this published article (and its supplementary information files). The datasets used and/or analyzed during the current study are available from the corresponding author on reasonable request.

\section{Declarations}

Ethical approval and consent to participate The review board of Persian Gulf University approved the study protocol. An online informed consent was obtained from all participants prior to their inclusion in the study. Those who agreed were permitted to complete a Web-based online questionnaire. The identities of the participants were not recorded to guarantee confidentiality. Furthermore, written permission to use the questionnaires has also been received by email from all authors.

Consent to publish Not Applicable

Competing interests The authors declare no competing interests.

\section{References}

Alzoubi H, Alnawaiseh N, Al-Mnayyis A, Lubad M, Aqel A, AlShagahin H (2020) COVID-19-knowledge, attitude and practice among medical and non-medical university students in Jordan. J Pure Appl Microbiol 14:17-24

Atchison CJ, Bowman L, Vrinten C, Redd R, Pristera P, Eaton JW, \& Ward H (2020) Perceptions and behavioural responses of the general public during the COVID-19 pandemic: a cross-sectional survey of UK Adults. MedRxiv

Bish A, Michie S (2010) Demographic and attitudinal determinants of protective behaviours during a pandemic: a review. Br J Health Psychol 15:797-824

Cai J, Sun W, Huang J, Gamber M, Wu J, He G (2020) Indirect virus transmission in cluster of COVID-19 cases, Wenzhou, China, 2020. Emerg Infect Dis 26:1343-1345

Chandrashekharayya S, Kavitha M, Prakash Handi PK, Ss D (2014) To study the level of awareness about complications of chronic suppurative otitis media $(\mathrm{CSOM})$ in CSOM patients. J Clin Diagn Res 8: 59

Das A. (2013). Information-seeking among pregnant women: a mixed method approach. (Doctor of Philosophy), Florida State University

De Zwart O, Veldhuijzen IK, Elam G, Aro AR, Abraham T, Bishop GD et al (2009) Perceived threat, risk perception, and efficacy beliefs related to SARS and other (emerging) infectious diseases: results of an international survey. Int J Behav Med 16:30-40

Ding Y, Du X, Li Q, Zhang M, Zhang Q, Tan X, Liu Q (2020) Risk perception of coronavirus disease 2019 (COVID-19) and its related factors among college students in China during quarantine. PLoS One 15:e237626

Esmaeilzadeh S, Ashrafi-Rizi H, Shahrzadi L, Mostafavi F (2018) A survey on adolescent health information seeking behavior related to high-risk behaviors in a selected educational district in Isfahan. PLoS One 13:e0206647

Faasse K, Newby J (2020) Public perceptions of COVID-19 in Australia: perceived risk, knowledge, health-protective behaviors, and vaccine intentions. Front Psychol 11:551004

Feinberg I, Greenberg D, \& Frijters J (2015) Understanding health information seeking behaviors of adults with low literacy, numeracy, and problem solving skills: results from the 2012 US PIAAC study. US PIAAC Study

Fung IC-H, Cairncross S (2007) How often do you wash your hands? A review of studies of hand-washing practices in the community 
during and after the SARS outbreak in 2003. Int J Environ Health Res 17:161-183

Glanz K, Bishop DB (2010) The role of behavioral science theory in development and implementation of public health interventions. Annu Rev Public Health 31:399-418

Hulin C, Netemeyer R, Cudeck R (2001) Can a reliability coefficient be too high? J Consum Psychol 10:55-58

Ishikawa H, Kato M, Kiuchi T (2016) Associations of health literacy and information sources with health-risk anxiety and protective behaviors. Journal of Communication in Healthcare 9:33-39

Jacobs W, Amuta AO, Jeon KC (2017) Health information seeking in the digital age: an analysis of health information seeking behavior among US adults. Cogent Soc Sci 3:1302785

Kolarić A, Stričević I (2017) Information seeking behavior for decision making in everyday life: a pilot study on adolescents. J Res Writing Books Cultural Heritage Institutions 9:275-308

Lambert SD, Loiselle CG (2007) Health information - seeking behavior. Qual Health Res 17:1006-1019

Lau JT, Griffiths S, Choi KC, Tsui HY (2009) Widespread public misconception in the early phase of the H1N1 influenza epidemic. J Infect 59:122-127

Lee K (2011) The role of media exposure, social exposure and biospheric value orientation in the environmental attitude-intention-behavior model in adolescents. J Environ Psychol 31:301-308

Liao Q, Cowling B, Lam WT, Ng MW, Fielding R (2010) Situational awareness and health protective responses to pandemic influenza A (H1N1) in Hong Kong: a cross-sectional study. PLoS One 5:e13350

Liu Y, Gayle AA, Wilder-Smith A, Rocklöv J (2020) The reproductive number of COVID-19 is higher compared to SARS coronavirus. J Travel Med:1-4

Mant M, Holland A, Prine A (2021) Canadian university students' perceptions of COVID-19 severity, susceptibility, and health behaviours during the early pandemic period. Public Health Res Pract 2: 100114

Marchionini G (1997) Information seeking in electronic environments: Cambridge university press

Namkoong K, Nah S, Record RA, Van Stee SK (2017) Communication, reasoning, and planned behaviors: unveiling the effect of interactive communication in an anti-smoking social media campaign. Health Commun 32:41-50

Peiris JS, Yuen KY, Osterhaus AD, Stöhr K (2003) The severe acute respiratory syndrome. N Engl J Med 349:2431-2441

Ramsey MA, Marczinski CA (2011) College students' perceptions of H1N1 flu risk and attitudes toward vaccination. Vaccine 29:7599 7601

Rosenstock IM (1974) Historical origins of the health belief model. Health Educ Monogr 2:328-335

Rosenstock IM, Strecher VJ, Becker MH (1988) Social learning theory and the health belief model. Health Educ Q 15:175-183

Rubin GJ, Amlôt R, Page L, Wessely S (2009) Public perceptions, anxiety, and behaviour change in relation to the swine flu outbreak: cross sectional telephone survey. BMJ 339:b2651

Shahin MH, Hussien RM (2020) Risk perception regarding the COVID19 outbreak among the general population: a comparative Middle East survey. Middle East Current Psychiatry 27:1-19

Shahnazi H, Ahmadi-Livani M, Pahlavanzadeh B, Rajabi A, Hamrah MS, Charkazi A (2020) Assessing preventive health behaviors from
COVID-19 based on the health belief model (HBM) among people in Golestan province: a cross-sectional study in Northern Iran. Infect Dis Poverty 9:157-165

Sheeran P, Harris PR, Epton T (2014) Does heightening risk appraisals change people's intentions and behavior? A meta-analysis of experimental studies. Psychol Bull 140:511-543

Sheppard J, Thomas CB (2021) Community pharmacists and communication in the time of COVID-19: applying the health belief model. Res Soc Adm Pharm 17:1984-1987

Shieh C, Broome ME, Stump TE (2010) Factors associated with health information-seeking in low-income pregnant women. Women Health 50:426-442

Tadese M, Haile AB, Moltot T, Silesh M (2021) Perceived risk of COVID-19 and related factors among university students in Ethiopia during school reopening. Infection and Drug Resistance 14:953-961

Taghrir MH, Borazjani R, Shiraly R (2020) COVID-19 and Iranian medical students; a survey on their related-knowledge, preventive behaviors and risk perception. Arch Iran Med 23:249-254

Teh CH, Teh MW, Lim KH, Kee CC, Sumarni MG, Heng PP et al (2019) Clustering of lifestyle risk behaviours and its determinants among school-going adolescents in a middle-income country: a crosssectional study. BMC Public Health 19:1-10

Venema TA, Pfattheicher S (2021) Perceived susceptibility to COVID-19 infection and narcissistic traits. Personal Individ Differ 175:110696

Wadood MA, Mamun A, Rafi MA, Kamrul Islam M, Mohd S, Lee LL, \& Hossain MG (2020) Knowledge, attitude, practice and perception regarding COVID-19 among students in Bangladesh: survey in Rajshahi University. Medrxiv

Wang Y, Wang Y, Chen Y, Qin Q (2020) Unique epidemiological and clinical features of the emerging 2019 novel coronavirus pneumonia (COVID-19) implicate special control measures. J Med Virol 92: $568-576$

Weinstein ND (2000) Perceived probability, perceived severity, and health-protective behavior. Health Psychol 19:65-74

Wickman ME, Anderson NLR, Greenberg CS (2008) The adolescent perception of invincibility and its influence on teen acceptance of health promotion strategies. J Pediatr Nurs 23:460-468

Wilson TD (2000) Human information behavior. Inf Sci 3:49-56

World Health Organization (2021a) Coronavirus disease (COVID-19) dashboard. World Health Organization Available from https:// covid19.who.int/

World Health Organization (2021b) Coronavirus disease (COVID-19) dashboard. World Health Organization. Available from https:// covid19.who.int/region/emro/country/ir

Yang Q, Wu S (2021) How social media exposure to health information influences Chinese people's health protective behavior during air pollution: a theory of planned behavior perspective. Health Commun 36:324-333

Yıldırım M, Güler A (2020) COVID-19 severity, self-efficacy, knowledge, preventive behaviors, and mental health in Turkey. Death Stud:1-8

Publisher's note Springer Nature remains neutral with regard to jurisdictional claims in published maps and institutional affiliations. 